\title{
Editorial Note: Machine Learning for Multimodal Data Understanding
}

\author{
Published online: 23 November 2018 \\ C Springer Science+Business Media, LLC, part of Springer Nature 2018
}

Multimedia Tools and Applications gratefully acknowledges the editorial work of the scholars listed below on the special issue entitled, "Machine Learning for Multimodal Data Understanding."

Of 56 papers submitted to this issue, 24 were eventually accepted after a stringent peer-review process.

\section{Weizhi Nie (Corresponding Guest Editor)}

Tianjin University, China

truman.nie@gmail.com

\section{Hanwang Zhang}

National University of Singapore, Singapore

hanwangzhang@gmail.com

\section{Fei Wu}

Zhejiang University, China

wufei@cs.zju.edu.cn

\section{Dr. Zhao-Zheng Yin}

Missouri University of Science and Technology, USA

yinz@mst.edu

Publisher's Note Springer Nature remains neutral with regard to jurisdictional claims in published maps and institutional affiliations. 